CULTOGRAPHIES 
CULTOGRAPHIES is a series of individual studies devoted to the analysis of cult film. The series provides a comprehensive introduction to those films that have attained the coveted status of a cult classic, focusing on their particular appeal, the ways in which they have been conceived, constructed, and received, and their place in the broader popular cultural landscape.

OTHER PUBLISHED TITLES IN THE CULTOGRAPHIES SERIES

THE ROCKY HORROR PICTURE SHOW

Jeffrey Weinstock

DONNIE DARKO

Geoff King

THIS IS SPINAL TAP

Ethan de Seife

BAD TASTE

Jim Barratt

SUPERSTAR:

THE KAREN CARPENTER STORY

Glyn Davis

BRING ME THE HEAD OF

ALFREDO GARCIA

Ian Cooper

BLADE RUNNER

Matt Hills

THE EVIL DEAD

Kate Egan

FASTER, PUSSYCAT! KILL! KILL!

Dean DeFino

QUADROPHENIA

Stephen Glynn
FRANKENSTEIN

Robert Horton

THEY LIVE

D. Harlan Wilson

DEEP RED

Alexia Kannas

MS. 45

Alexandra Heller-Nicholas

STRANGER THAN PARADISE

Jamie Sexton

THE HOLY MOUNTAIN

Alessandra Santos

SERENITY

Frederick Blichert

DANGER: DIABOLIK

Leon Hunt

I SPIT ON YOUR GRAVE

David Maguire

THE SHINING

Kevin J. Donnelly 


\section{FLAMING CREATURES}

Constantine Verevis

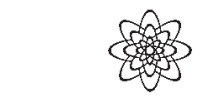

WALLFLOWER PRESS

NEW YORK 
A Wallflower Book

Published by

Columbia University Press

Publishers Since 1893

New York • Chichester, West Sussex

cup.columbia.edu

Copyright (c) 2020 Columbia University Press

All rights reserved

Library of Congress Cataloging-in-Publication Data

Names: Verevis, Constantine, author.

Title: Flaming creatures / Constantine Verevis.

Description: London ; New York: Wallflower Press, 2019. | Series:

Cultographies | Includes bibliographical references and index.

Identifiers: LCCN 2019025481 (print) | LCCN 2019025482 (ebook) |

ISBN 9780231191470 (paperback) | ISBN 9780231851305 (ebook)

Subjects: LCSH: Flaming creatures (Motion picture) |

Experimental films-History and criticism.

Classification: LCC PN1997.F54 V47 2019 (print) |

LCC PN1997.F54 (ebook) | DDC 791.43/72—dc23

LC record available at https://lccn.loc.gov/2019025481

LC ebook record available at https://lccn.loc.gov/2019025482

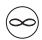

Series design by Elsa Mathern

Cover image: Jack Smith, Flaming Creatures, 1962-63, film still from $16 \mathrm{~mm}$ black and white release print.

(c) Jack Smith Archive. Courtesy of Gladstone Gallery, New York and Brussels. 